\title{
IntuPaint: Bridging the Gap between Physical and Digital Painting
}

\author{
Peter Vandoren, Tom Van Laerhoven, Luc Claesen, \\ Johannes Taelman, Chris Raymaekers and Frank Van Reeth \\ Hasselt University - $t U L$ - IBBT \\ Expertise Centre for Digital Media \\ 3590 Diepenbeek, Belgium \\ \{firstname.lastname\}@uhasselt.be
}

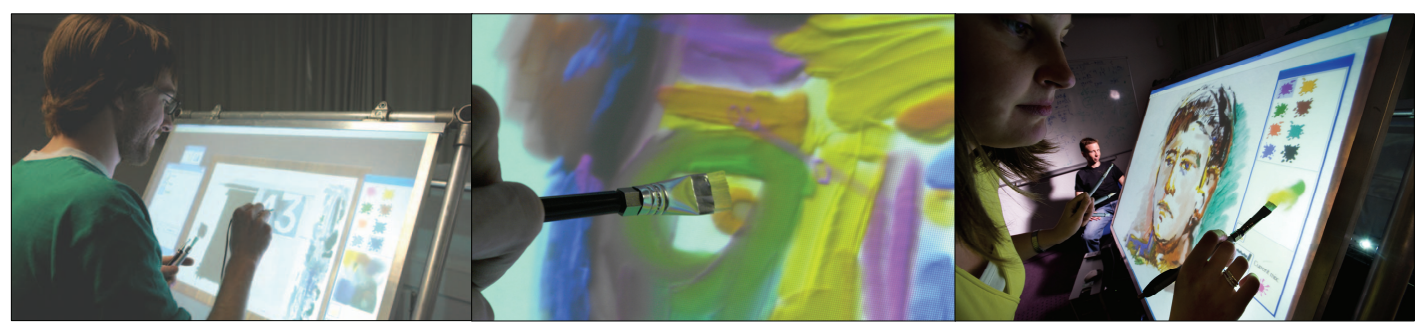

\begin{abstract}
This paper presents a novel interface for a digital paint system: IntuPaint. A tangible interface for a digital paint easel, using an interactive surface and electronic brushes with a tuft of bristles, has been developed. The flexible brush bristles conduct light by means of total internal reflection inside the individual bristles. This enables to capture subtle paint nuances of the artist in a way that was not possible in previous technologies. This approach provides natural interaction and enables detailed tracking of specific brush strokes. Additional tangible and finger-based input techniques allow for specific paint operations or effects. IntuPaint also offers an extensive model-based paint simulation, rendering realistic paint results. The reality-based approach in the combination of user interface and paint software is a new step forward to bridge the gap between physical and digital painting, as is demonstrated by initial user tests.
\end{abstract}

\section{Introduction}

Digital paint systems have evolved a lot in the past years [20]. The paint applications provide a large gallery of paint functions, numerous settings for brushes, paints, layers, ... and have options to digitally augment the painting process. Although digital painting offers several advantages (e.g., information saving, stability, no paint dry time, undo paint strokes), many painters do not even try out digital painting. Even stronger, they are convinced that physical (also called traditional) painting and digital painting will remain two separate worlds, having its own advantages and disadvantages. A large part of this vision is situated in the considerable differences in user experience, resulting in a wide gap between both paint environments.

Digital paint systems have been using separate input devices and display devices for quite some time. The use of a dedicated input tablet (e.g., Wacom tablets [25]) leads to eye-hand coordination problems that, however, can be overcome by practice. The current stiff digital styluses can in software be attributed with brush-like functionalities, but fall short in the actual user experience. The rude (if any) force feedback, the absence of the bending of the brush tuft, the lack of friction, the point-based input and the visual absence of the (correct) brush shape remain strong points of concern and thus lead to additional cognitive load for the user. A lot of professional digital painting systems are also overloaded with functionalities, as painter $\mathrm{J}$. Faure Walker [5] states: "Adobe PhotoShop is the equivalent of a Boeing 747: a brilliant functional artifact, too complex for a single human mind to comprehend". And in spite of this complexity, most paint systems offer limited paint simulation models, leading to unexpected colors and paint results.

This paper presents a novel interface for a digital paint system, called IntuPaint. IntuPaint is a hybrid platform, integrating important characteristics of 
traditional painting into a comprehensive paint simulation environment. The infrared light conducting bristle fibers of the novel paint brushes are the key innovation in the palette of tangible paint instruments. The setup of the interactive paint surface like a digital paint easel provides for natural interaction, reinforcing embodied interaction and enabling simultaneous interaction. The highly detailed, vision-based tracking of the infrared brush complements the extensive paint simulation, jointly leveraging the intuitive interaction by generating highly realistic paint results. As a result, IntuPaint allows the artist to focus on the actual artwork. IntuPaint is therefore the first platform that offers a solution to the majority of the abovementioned shortcomings of digital painting.

\section{System architecture}

The structure of the IntuPaint platform is shown in figure 1. In the next paragraphs we will briefly describe the major components of the system.

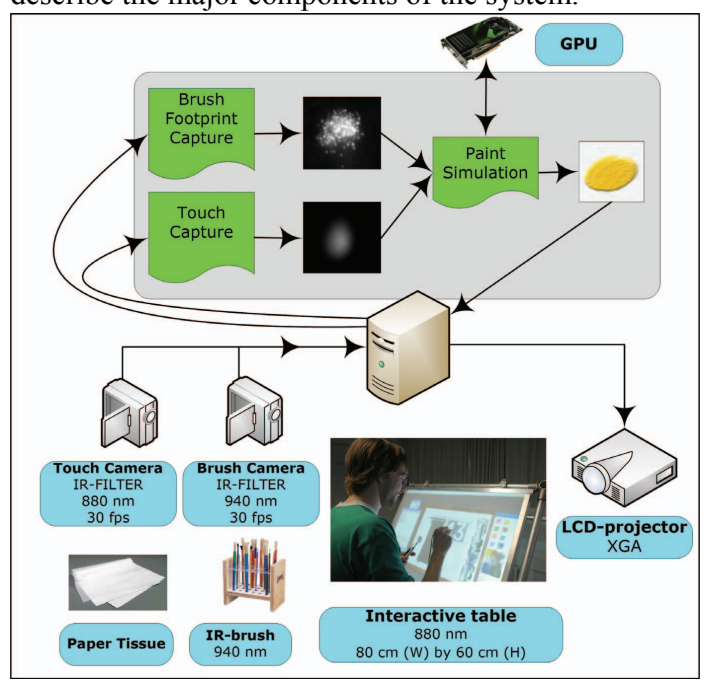

Figure 1. Architectural overview of IntuPaint.

The paint table is an interactive surface based on the principle of frustrated total internal reflection (FTIR) [7]. The table has an optical diffuse film surface that serves for projecting the image of the rearplaced off-the-shelf LCD-projector. Touch input on the table is recorded by a monochrome 0.3 megapixel video camera, equipped with an IR band-pass filter at 880 nanometer (nm). We tilted the table about 70 degrees, to approach a paint easel setup.

The paint brushes [22] are equipped with an IR-led, mounted inside the shaft of the brush (figure 3) and operating at $940 \mathrm{~nm}$. The bristles of the brushes are flexible and transparent nylon fibers, available in different qualities and thicknesses. Each of the transparent bristles in the tuft of the brush, conduct infrared light by means of total internal reflection. The infrared light leaves the bristles at the end points. If the bristles are a little bit structured on the surface, light is also emitted on that surface. The use of ferrules allows customizing the brush tuft shape. The contact surface of the brush on the table surface is tracked by a 2 megapixel video camera, equipped with a band-pass filter at $940 \mathrm{~nm}$.

A secondary input medium is a paper tissue, which can be used to remove paint from the paint canvas.

The paint simulation [23, 24] is the software cornerstone of the IntuPaint platform. It uses physically-based and empirically-based algorithms to simulate the complex behavior of paint media like watercolor, gouache and impasto. Simulation of the drying process, bi-directional paint transfer between brush and canvas, and a specialized color mixing scheme are vital elements to obtain a "natural look" in the resulting images. In this paper we chose to work mainly with impasto paint for its appealing textured, almost three-dimensional appearance.

IntuPaint runs on a recent desktop PC (Intel Core2, 2 GB RAM, Windows XP) equipped with a recent graphics card (nVidia GeForce $8800 \mathrm{GT}$ ), since the paint simulation relies on programmable graphics hardware (GPU) to get the required performance.

\section{From digital brush to paint stroke}

\subsection{A new digital brush design}

One of the key elements in the painting experience is the brush, and in particular the handling and the shape of the brush. It is the contact surface of the brushes' bristles with the paint canvas (the so-called footprint of the brush) that actually shapes the paint stroke. The footprint takes different forms depending on pressure, angle to the canvas, rotation of the brush, speed of movement. A clear view and a precise control over the brush and its bristles is therefore of utmost importance to the painter, especially when learning to work with a new paint environment. It is also important to accurately register this footprint, in order to render realistic paint strokes and effects.

Hence digital painting using a stylus with a stiff tip is not that intuitive, because the user is not able to see the footprint. Adding an artificial visualization of the virtual brush tip on the painting canvas merely causes confusion once the stylus is (slightly) pressured onto the canvas (see fig. 2). Systems that use a brush 
equipped with a stiff stylus position determination system, such as e.g., in an electromagnetic tablet system, need to infer the tuft's shape and movement only from the position of the stylus, leading to undesired effects as shown in figure 2. As for the systems that use a real brush without actual footprint tracking, they need to simulate the bristles of a brush and hence introduce inaccuracy and confuse the user.

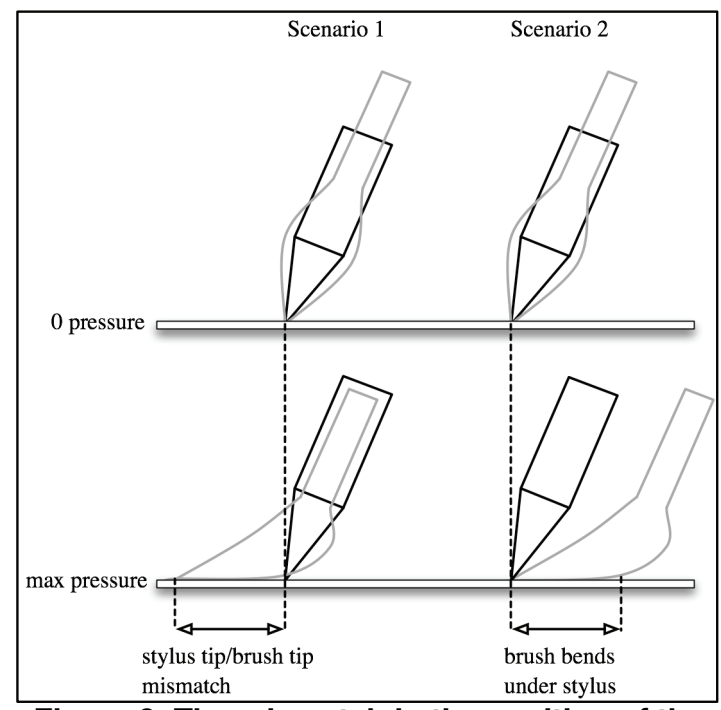

Figure 2. The mismatch in the position of the stylus with regard to its visualization.

Although some technologies do exist that allow for brush tracking $[3,10,12,14,19]$ or footprint capturing $[8,11,15,16,18]$, we think these systems do not allow precise tracking of the brushes' footprint or are not really suitable for tabletop environments, as is elaborated in paragraph 6 on related work. This is the basis for the design of the infrared (IR) light emitting brush as presented in this paper.

The bristles of the IR-brush (figure 3) are lightconducting nylon fibers, which are available in different qualities and thicknesses. We developed early brush prototypes with fiber thickness of about 500 micrometer $(\mu \mathrm{m})$, as a proof-of-concept for the detailed footprint tracking. Because of the lack of flexibility of these thicker fibers and thus rather poor handling, we shifted gradually to thinner fibers, ending up with a thickness of $40 \mu \mathrm{m}$. We also experimented with fibers with regard to lateral light emission, since side contacts of the brush with the canvas are also to be registered.

Finally we experimented with different fiber strengths, which influence the tactile qualities of the brush. In the end, we have developed a set of brushes, having various tuft shapes and implemented using various fiber types.

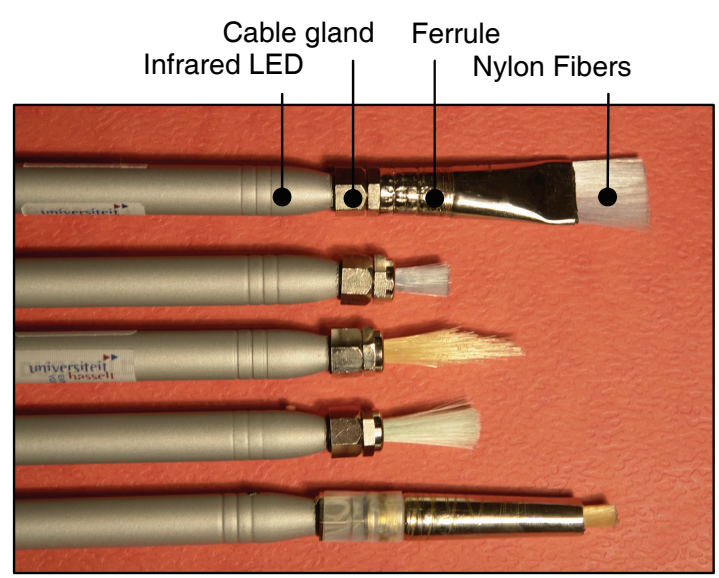

Figure 3. The IR-brushes

Our first prototypes required low power. However, user experience tests demonstrated that it is rather difficult to hold a number of tethered brushes in the non-dominant hand without the respective power cables getting twisted. So we decided to go for a cordless, battery-powered version. We installed a push-button on the brush body, enabling power consumption control by explicit brush activation.

\subsection{Capturing the footprint of the IR-brush}

The quality of the footprint tracking is important to the usability of IntuPaint. We made an initial setup for tracking the tuft footprint by employing a modest video camera (0.3 megapixel), placed at a small distance behind the canvas and operating at 60 frames per second. The tip of the IR-brush was oval shaped and was made of thicker bristles (fibers of $400 \mu \mathrm{m}$ diameter). Figure 4 shows some downscaled (about $25 \%$ ) snapshots of the contact area of the IR-brush. We identified three key elements in our tracking implementation: realism of the footprint, resolution of the footprint and real-time processing.

Figure 4.a shows the contact surface of the brush as seen from behind the diffuse surface by the infrared camera. This figure demonstrates the feasibility of the IR-light tracking on the canvas and shows the resolution of the tracked footprint. Using simple image processing techniques (background subtraction, spatial filtering, and segmentation) we transform the footprint to a 64 by 64 pixel bitmap consisting of brightness values ranging from 0 to 255 . This bitmap is then transferred to our paint simulation software for further processing and rendering. 

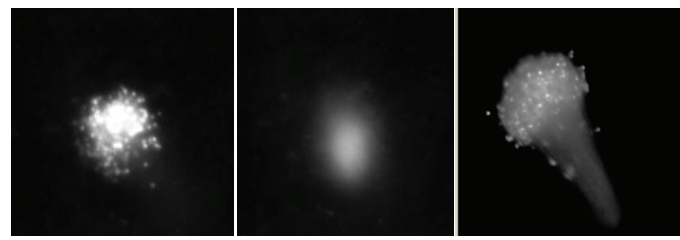

Figure 4.a. The contact surface of the IR-brush on the canvas (left). 4.b. IR-light on the canvas when the brush is near the canvas (middle).

4.c. The side contact surface of the IR-brush when pressed on the canvas (right).

When the brush is very near to the canvas, the fibers emit light on the canvas (figure 4.b). Because the brightness level of this light is rather low, we managed to differentiate this situation from real paint actions (when the brush actually touches the canvas). Another important element is tracking the side surface of the brush, because exerting pressure on a brush causes the side surface of bristles to touch the canvas. As the IR-light also leaves the individual fibers sideways, a realistic brush footprint can be tracked. The side contact surface is identified by the conic shape with lower brightness (figure 4.c).

Real-time processing is in a trade-off position with several environment variables e.g., the degree of realism in the paint simulation software, the size of the drawing canvas, the frequency of the footprint tracking and the resolution of this tracking. Our current design focuses on reality-based interaction [9] and simulation, although we gradually introduced optimizations to increase the responsiveness of the paint system.

\section{Interaction in IntuPaint}

\subsection{User interface}

The visual part of the IntuPaint user interface (figure 5) is modeled according to the center stage design [21] and consists of three areas: the paint canvas (central), the paint toolbox (right) and advanced simulation options (left). In the task oriented user interface of IntuPaint, each area does offer a specific functionality and has its preferred input channels.

The paint canvas is centrally located on the interactive table, and measures about $40 \mathrm{~cm}$ wide by $30 \mathrm{~cm}$ high. This area allows using a brush (painting), a finger (to distort paint) or a paper tissue (erase paint). The erase function gradually removes paint from the canvas. The paint canvas is visualized inside a wooden frame, representing the canvas manipulation area. The user can translate and rotate the paint area, by placing one or two fingers on the wooden frame and subsequently gesturing to reach the desired position and orientation.

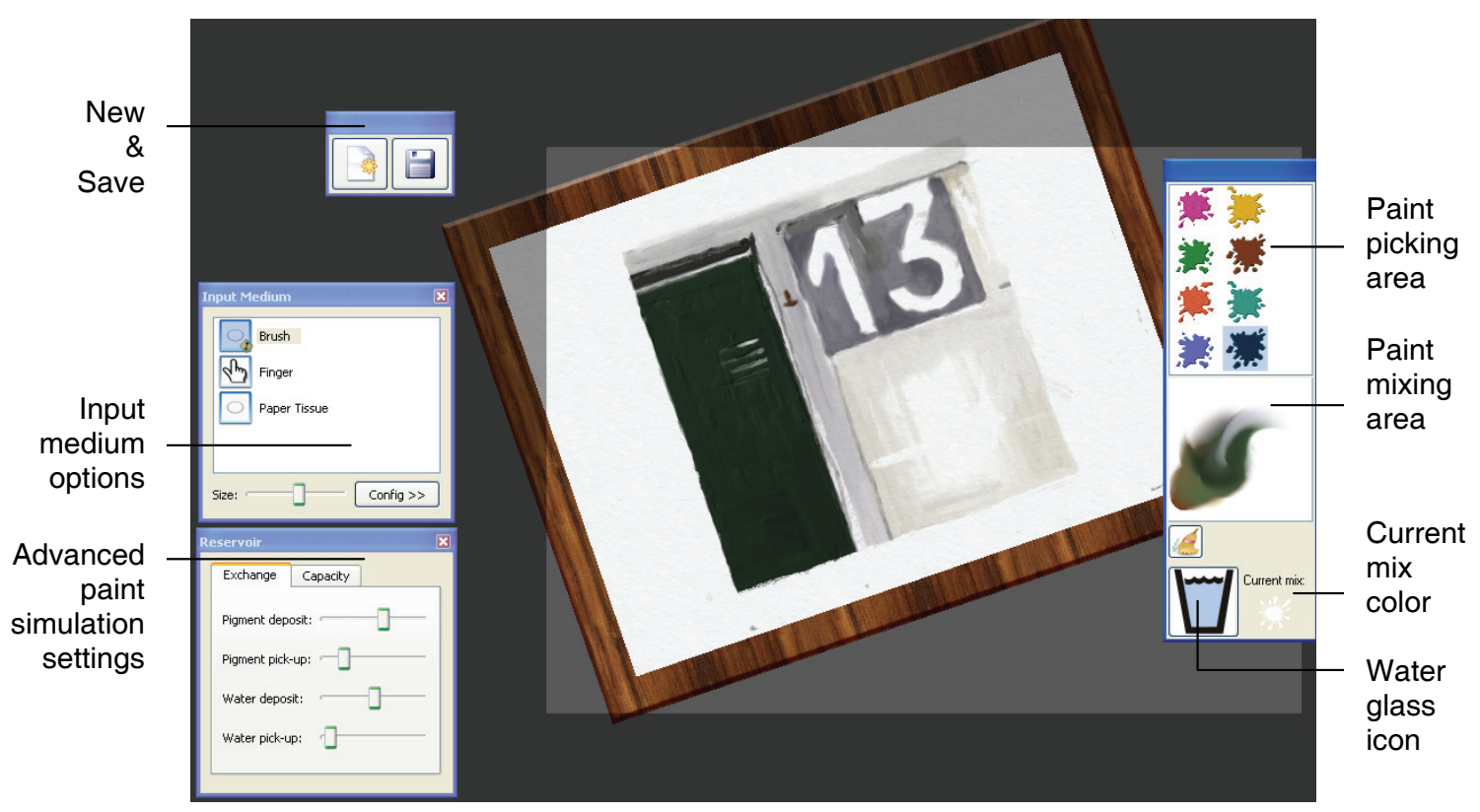

Figure 5. The UI widgets of IntuPaint (for right-handed users) 
The brighter, rectangular area in the background texture of the interactive table symbolizes the brushes' activity zone, and limits the area in which the user can use the brush. This zone concept came into place as a trade-off between computational speed of the paint simulation and size/resolution of the painting. When parts of the canvas move outside this zone, we provide a shadow-like effect on those parts; this effect however causes no difficulties with regard to the human color perception [26].

The right hand side of figure 5 shows an overview of the available paint selection and mixing options. Dipping the brush in one of the eight paint colors loads a preset amount of paint. A dedicated area allows for mixing paint until the desired color is obtained. The actual color of the paint in the brush is reflected by the paint splash, displayed in the lower area on the right. The brush or the user's fingers can be cleaned by 'dipping' them into the glass of water.

The left area of the user interface is reserved for more advanced painting options. IntuPaint uses a pigment model to simulate the pigment deposit and pick-up. The amounts of pigment transfer can be preset using the top two sliders in the 'reservoir' box. The simulation of water deposit and pick-up is applicable to water-based paint materials (e.g., aquarelle). The current pigment locations in the brush are also accounted for and can be consulted by selecting the 'capacity'-tab with a finger. The sliders can be repositioned with a finger, altering paint parameters while painting and leading to special effects. These options were suggested to us by some professional painters, who liked to exploit the extra possibilities of digital painting. The info in the reservoir-box is specific to the input medium, which can be selected in the 'input medium' box. In the upper left area of the interface we also provide options for clearing the paint canvas and for saving the current painting.

\subsection{Integrated interaction concepts}

IntuPaint tries to bridge the gap between physical painting and digital painting by offering a paint environment that resembles a paint easel setup. The input devices share form factors with the traditional paint instruments and have a nearly identical function as in the real world. The setup therefore provides for embodied interaction [4] and should also allow easy skills transfer [17].

The user interface was designed for right-handed users. The paint options reside on the right hand side of the interactive table, avoiding arms crossing on actions from the non-dominant hand. The user interface allows for asymmetric bimanual interaction, designed according to the three principles listed by Guiard [6]. The (fingers of the) non-dominant hand could rotate and/or translate the canvas while the dominant hand holds the brush. Another example is the use of finger input with the non-dominant hand to disperse some paint, which precedes overpainting the area with the brush held by the dominant hand. The dominant hand performs the most precise actions, painting with the brush and carefully removing paint with the tissue.

In their recent work on reality-based interaction, Jacob et al. [9] mention that a useful interface can rarely entirely mimic the real world, but will necessarily include some unrealistic or artificial features and commands. The authors advise interface designers therefore to strike a balance between the level of reality of the interface and its power. IntuPaint incorporates some trade-offs, e.g., the rotatable canvas, the water glass-icon, the push-button on the batteryoperated IR-brush.

\section{User experiences}

Our target audience is twofold. IntuPaint aims primarily at the traditional painters, which stick to physical painting after possibly (or not) having had a go at digital painting. In second place, IntuPaint could appeal to the current users of digital paint platforms, but seeking solutions for the disadvantages of the present digital systems. Although we have had discussions and small individual tests with amateur and professional painters, we decided to organize an informal user test to gain insights on the user experience of a group of artists that were new to IntuPaint.

\subsection{Setup}

Six persons participated in the user evaluation session. Test participants varied in age from 25 to 60 , and were all right-handed. Three persons were mainly active in physical painting, while the others were more experienced in creating digital artwork. Five out of six people were familiar with using computers, although four of them never worked with a multitouch surface; the sixth person, also the eldest participant, had no computer experience at all. All test users had an explicit interest in painting and creative design, as three were actual amateur painters. One test person had already worked with an earlier version of IntuPaint.

The test session lasted about one hour per participant. It included an explanation of the paint environment, a trial paint session starting from a 
template drawing (10 minutes) and half an hour work painting on a subject of choice. Afterwards we asked our artists to fill out a user questionnaire, with openended questions as well as closed-ended questions. The test session was concluded by a small debriefing session (10 minutes).

The test participants had three IR-brushes at their disposal: a flat brush, a small round brush and a medium-sized round brush. The paint type was impasto. We defined a rather high paint deposit, which enabled enriching the painting.

\subsection{Results}

The test users were unanimous about the fact that the user interface of IntuPaint was intuitive; all test subjects started to paint quite easily and quite seldom asked for additional information while painting.

They were all positively surprised by the design of the brushes. Some of the participants had expected stylus-based input devices instead of electronic brushes with bristles and tuft. Most of the test users asked for additional brushes: larger types (for initially covering larger parts of the canvas) as well as very fine types (for details). One participant suggested adding a pencil to the brush collection for very detailed painting. All users indicated they appreciated seeing the actual bristles of the paint brush, since it facilitated drawing.

Two persons indicated that some of the form factors of a real brush were missing (e.g., length of the stylus and friction in case of painting with high viscosity paints like impasto), although they did not indicate that it bothered them during the test. Half of the test group mentioned that, after painting for while, their initial perception of painting with special electronic brushes disappeared and they started to perceive the digital brush as a real brush.

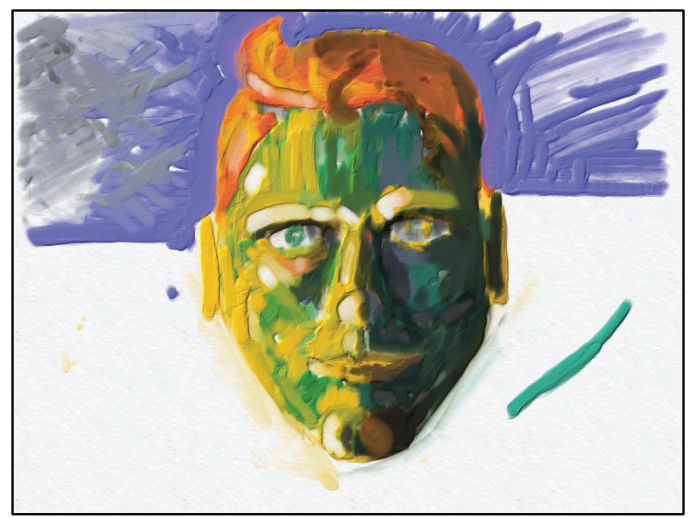

All except one user liked the finger interaction for spreading paint over the canvas. The paper tissue feature was also appreciated. Four test users found the painting canvas to be sufficient in size, while the other two users judged the canvas to be a bit small.

The paint rendering process was judged to be a bit slow by one half of the test population. However, none of these test subjects actually found the delay, between painting the stroke and the corresponding stroke visualization on the canvas, disturbing. The users noticed the delay when painting several longer strokes in a short sequence (e.g., painting the background). The actual paint results rendered by the paint simulation were positively evaluated: only one participant indicated that the paint simulation could be enhanced in a specific situation.

Our test persons were rather neutral in the approval of their paintings (see figure 6 for some paintings) and were quite unanimous about wanting to have more time to actually further develop their painting.

One of the test users, an experienced amateur painter swearing by physical painting, mentioned that he would prefer IntuPaint to other digital paint platforms when precompositioning future paintings.

All except two test subjects suggested an undofeature as an interesting extension. The majority of the test group also suggested integrating a zooming feature.

\subsection{Discussion}

Although the test session was rather short, we can say that the participants validated the intuitiveness of the paint environment. The paintings by the participants (presented on the first page of the paper as well in figure 6) illustrate that IntuPaint does allow a painter to express the artistic ideas on the canvas.

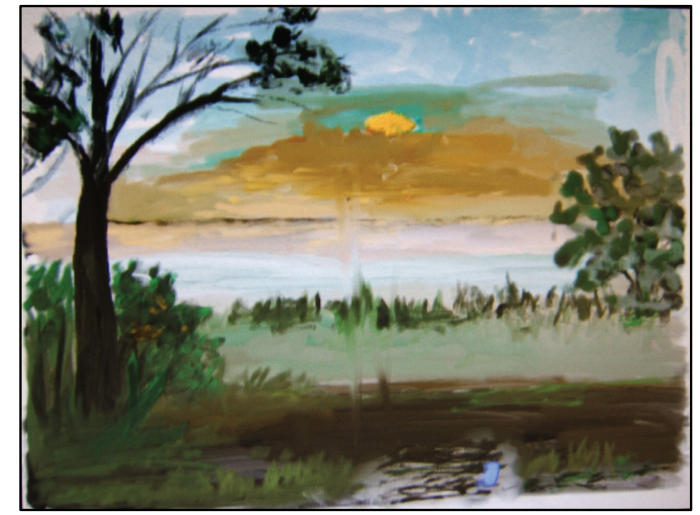

Figure 6. Selected artworks of the test participants, made with IntuPaint in about $\mathbf{3 0}$ minutes using impasto paint. 
The remarks about the differences in handling between the digital and the physical brushes are of course correct. The initial prototypes were tethered, and retrieved the power for the LED externally. By integrating a small battery in the brush we added some weight, which shifted the center of gravity of the brushes outward. Although we managed to introduce a realistic haptic feeling by using bristles, test participants missed the paint friction when using high viscosity paint like impasto (e.g., when overpainting or mixing paint); we think that when using low viscosity paint like waterpaint, this would be a smaller issue. However, the test did also show that the behavior of the digital paint brushes sufficiently approached that of a real brush; half of the participants perceived the painting action with bendable bristles in the brush tuft and the real-time visualization of the painting result as quite real. We suppose the reality-based interaction and the task oriented interface also played a part in this perception. The participant without computer experience actually demonstrated the possibly immersive nature of IntuPaint: when cleaning the paint brush, the person 'tapped' the water glass icon several times, resembling the dipping movement of a real brush in a real glass filled with water when using watery paint.

The mentioned latency in the system response is largely influenced by the complexity of the paint simulation. The delay came to the attention of the users after the sequence of consecutive paint strokes, e.g., for the background of the painting. When designing IntuPaint, we made decisions with regard to the tradeoff between accuracy of tracking and simulation vs. responsiveness of the system. We have given priority to the high-resolution registration of brush interaction and the comprehensive, realistic simulation, and subsequently introduced step by step optimizations or simplifications to improve responsiveness.

The neutral evaluation of their proper painting could be related to the fact that all artists wanted more time to work. Knowing that making a painting requires days, weeks or even months, we realize that it could have been frustrating to the test users having to end the creative process after a very short time.

\section{Related work}

Simulation of a paint brush in digital painting can be achieved by working with software models and algorithms, an alternative input device or the combination of both. Each approach has its advantages and limitations, which have to be related to the envisioned research objectives.
Algorithms allow for model based approximation of the contact surface of a brush with the paint surface. Determining the footprint of a brush however is crucial to a realistic simulation of digital painting. CoolPaint [12] uses a simplified model, but acknowledges its shortcomings in the degree of realism in the paint simulation. Baxter et al. [1] used a complex 3D geometric, physically-based model of a brush, as did $\mathrm{Chu}$ [3] and $\mathrm{Xu}[26]$.

All kinds of brush equivalents are applied in digital paint simulations, depending on the desired degree of detail/realism in painting and the intended target audience. Finger-based painting is used on interactive surfaces like e.g., HabilisDraw DT [2] and Microsoft Surface [13], allowing for intuitive and easy but rather elementary drawing/painting. Off-the-shelf brushes are equipped with tracking technology, ranging from ultrasonic/infrared tracking [3] to inertial trackers [12, $3,10]$.

Other approaches opted for an augmented brush or a brush-like design. Nakakoji et al. developed a digital learning environment for Japanese calligraphy [14], using a modified brush, which position is tracked by a MERL DiamondTouch table. The I/O Brush [19], an oversized brush tracked by a Wacom Cintiq [25], uses a camera-captured color or image to paint.

Vision techniques can be applied to identify the footprint of traditional paint brushes. The diffuse IRillumination used in Microsoft Surface [13] does allow for rather detailed footprint detection, but is to our opinion not ideally suited for high-detailed tracking because of the light diffusion technique. Digital Canvas [15] works with the shadow detection of the brush on the paint canvas; shadows of hands, brush stylus and other objects are however difficult to discriminate from the actual brush footprint. ThermoTablet [8] detects touch regions of physical input objects on a interactive surface using temperature changes. Han [9] developed an interactive table using FTIR with a diffuser screen and a compliant surface layer, in order to combine tracking with a projected display but without defocusing of the tracking. These interactive touch tables have a pressure threshold that is too high for detecting the required nuances of soft brush strokes.

Rozin [18] and Knörig [11] integrated infrared light in a rather large brush, rendering a large round IR-light spot on the canvas that was used for layered videopainting or for music composing by painting. Piazza [16] used the light spot of a light emitting pen as an elementary paint brush.

The abovementioned systems differ in one or more aspects from IntuPaint. Our platform is designed with coinciding action and perception spaces and provides 
innovative, parallel input channels. The tangible, intuitive and accurate input media in combination with the high-end paint simulation software articulate the unique reality-based approach of IntuPaint.

\section{Conclusions and Future work}

In this paper we presented a digital paint platform that combines reality-based input techniques and devices with an extensive paint simulation. A user experience test acknowledges the intuitive nature and the expressional possibilities of the environment, therefore allowing bridging the gap between physical and digital painting.

Further research will be performed on comparing user experiences with experiences on stylus-based paint environments. Additional progression in the design of the IR-brushes would also enhance user experience and control. In this context we also plan speeding up the responsiveness of the paint system.

\section{Acknowledgements}

The authors would like to thank the test users for their valuable feedback and Karel Robert for his crucial contribution in the making of the accompanying video. Part of the research at EDM is funded by the ERDF (European Regional Development Fund) and the Flemish government.

\section{References}

[1] Baxter,W., Scheib, V., Lin, M., and Manocha, D. DAB: Haptic painting with 3D virtual brushes. In Proc. SIGGRAPH 2001, ACM Press (2001), 461-468

[2] Butler, C. G., and St. Amant, R. 2004. HabilisDraw DT: a bimanual tool-based direct manipulation drawing environment. In CHI '04 Extended Abstracts on Human Factors in Computing Systems (Vienna, Austria, April 24 29, 2004). CHI '04. ACM, New York, NY, 1301-1304.

[3] Chu, N., and Tai, C.-L.. Real-Time Painting with an Expressive Virtual Chinese Brush, IEEE Computer Graphics and Applications, 24, 5 (2004), 76-85.

[4] Dourish, P. Where The Action Is: The Foundations of Embodied Interaction, MIT Press, Cambridge, Mass., 2001.

[5] Faure Walker, J. Painting the Digital River - How an Artist Learned to Love the Computer, Prentice Hall, 2006, ISBN 0-13-173902-6

[6] Guiard, Y. Asymmetric division of labour in human skilled bimanual action: The kinematic chain as a model. Journal of Motor Behaviour, 19(1987), 486-517.

[7] Han, J. Y. Low-cost multi-touch sensing through frustrated total internal reflection. In Proc. UIST 2005, ACM Press (2005), 115-118.
[8] Iwai, D. and Sato, K. Heat sensation in image creation with thermal vision. In Proc. ACE '05. ACM, NY, 213-216.

[9] Jacob, R. J., Girouard, A., Hirshfield, L. M., Horn, M. S., Shaer, O., Solovey, E. T., and Zigelbaum, J. RealityBased Interaction: A Framework for POST-WIMP Interfaces. Proc. ACM CHI 2008 Human Factors in Computing Systems Conference, pp. 201-210, ACM Press (2008)

[10] Keefe, D. F., Feliz, D. A., Moscovich, T., Laidlaw, D. H., and LaViola, J. J. 2001. CavePainting: a fully immersive 3D artistic medium and interactive experience. In Proc. Symposium on interactive 3D Graphics I3D '01, 85-93.

[11] Knörig, A., Müller, B., and Wettach, R. Articulated Paint: Musical Expression for Non-Musicians. In Proc. New Interfaces for Musical Expression (NIME) 2007, 384-385

[12] Lang, D., Findlater, L., and Shaver, M., CoolPaint: Direct Interaction Painting, In Proc. UIST 2003, Canada, Poster

[13] Microsoft Surface, http://www.microsoft.com/surface/ [14] Nakakoji, K., Jo, K., Yamamoto, Y., Nishinaka, Y., and Asada, M. Reproducing and Re-experiencing the Writing Process in Japanese Calligraphy. In Proc. IEEE TableTop 2007, 75-78

[15] Park, J., Digital Canvas: A Projection-Space Interaction Tool. In Proc. Edutainment 2006, Lecture Notes in Computer Science (LNCS), 3942, 1171-1179

[16] Piazza, T., and Fjeld, M. Ortholumen: Using Light for Direct Tabletop Input. In Proc. IEEE TableTop 2007, 1-4

[17] Reed, S.K., Transfer on trial: Intelligence, Cognition and Instruction. In Singley, K. and Anderson, J.R. Eds, The Transfer of Cognitive Skill, Harvard University Press, Cambridge, MA, 1989, 39

[18] Rozin, D., http://www.smoothware.com/danny/ neweasel. html

[19] Ryokai, K., Marti, S., and Ishii, H. I/O Brush: Drawing with Everyday Objects as Ink. In Proc. CHI 2004, 303-310

[20] Smith A.R., "Digital Paint Systems: An Anecdotal and Historical Overview", IEEE Annals of the History of Computing, 23, 2(2001), 4-30.

[21] Tidwell, J. Designing Interfaces. Patterns for Effective Interaction Design, O'Reilly, 2005, ISBN 0-596-00803-1

[22] Vandoren, P., Van Laerhoven, T., Claesen, L., Taelman, J., Di Fiore, F., Van Reeth, F., and Flerackers, E. DIP-IT: Digital Infrared Painting on an Interactive Table. In CHI 2008 Extended Abstracts, 2901 - 2906, ACM, ISBN 978-1-60558-012-X, Apr. 2008

[23] Van Laerhoven, T., and Van Reeth, F. Real-time Simulation of Watery Paint, Computer Animation and Virtual Worlds, 16, 3-4 (2004), 429-439

[24] Van Laerhoven, T., Liesenborgs, J., and Van Reeth, F. Real-time Watercolor Painting on a Distributed Paper Model. In Proc. Computer Graphics International (CGI) 2004, 640643

[25] Wacom, http://www.wacom.com/

[26] Werner, J. S., Pinna, B., and Spillmann, L. Illusory Color \& the Brain, Scientific American, March 2007, 90-95

[27] Xu, S., Lau, F., Tang, F., and Pan, Y. Advanced Design for a Realistic Virtual Brush, Computer Graphics Forum, 22, 3 (2003), 533-542 